\title{
Isolation and Culture of Individual Myofibers and Their Adjacent Muscle Stem Cells from Aged and Adult Skeletal Muscle
}

\author{
Sören S. Hüttner, Hellen E. Ahrens, Manuel Schmidt, Henriette Henze, \\ Marie Juliane Jung, Svenja C. Schüler, and Julia von Maltzahn
}

\begin{abstract}
The isolation and culture of single floating myofibers with their adjacent muscle stem cells allow the analysis and comparison of muscle stem cells from aged and young mice. This method has the advantage that muscle stem cells are cultured on the myofiber, thereby culturing them in conditions as close to their endogenous niche as possible. Here we describe the isolation, culture, transfection with siRNA, and subsequent immunostaining for muscle stem cells on their adjacent myofibers from aged and young mice.
\end{abstract}

Keywords Muscle stem cell, Myofiber, Aging, Self-renewal, Differentiation, Collagenase, Transfection, Satellite cell

\section{Introduction}

Regeneration of skeletal muscle in the adult is carried out by muscle stem cells (MuSCs), also called satellite cells [1,2]. MuSCs in the adult are located between the basal lamina and the sarcolemma of a myofiber and are characterized by the expression of the transcription factor Pax7 [3]. Under normal resting conditions, MuSCs are quiescent and also express Sproutyl [4]. Following injury of the muscle or due to other stimuli, they get activated [5]. After activation MuSCs differentiate into myoblasts is accompanied by the expression of MyoD, which then further differentiate into myotubes and mature into myofibers, the contractile units of skeletal muscle $[5,6]$.

The dysfunction of MuSCs during aging is a major contributor to the decreased regenerative capacity of aged skeletal muscle. Multiple signaling pathways are upregulated in aged muscle stem cells such as JAK/STAT or p38 signaling [7-9]. Furthermore aged or geriatric MuSCs are characterized by entering a pre-senescence state and aberrant expression of Hoxa9 following activation $[10,11]$. 
Since muscle stem cells lose their stem cell properties and differentiate into myoblasts when cultured directly on cell culture plates, the functionality of muscle stem cells can only be investigated in vivo or in vitro by using the single floating myofiber culture system, where MuSCs are cultured on their adjacent myofiber. MuSCs on myofibers can then be analyzed for the expression of different myogenic markers such as Pax7 and MyoD to determine their state of differentiation. Quiescent as well as self-renewing MuSCs are characterized by the expression of Pax7, while proliferating MuSCs express Pax7 and MyoD, and early differentiating cells like myoblasts are only positive for MyoD. After $42 \mathrm{~h}$ of culture on their adjacent myofibers MuSCs have divided once, divisions are oriented either apical-basal or planar [6]. Multicellular clusters are formed after $72 \mathrm{~h}$ of culture consisting of self-renewing MuSCs, proliferating MuSCs, and further differentiated cells; each cluster is formed by a single MuSC [3, 6, 12]. The composition of a cluster can be analyzed for the number or percentages of Pax7 only, Pax7/MyoD double, and MyoD only positive cells to investigate the ability to self-renew, to proliferate, and to differentiate. During aging the numbers of MuSCs are decreased $[8,11]$. Furthermore, MuSCs from aged mice have a reduced ability to activate and proliferate $[8,10,11]$.

Investigation of the influence of signaling pathways or specific proteins on the functionality of MuSCs on their adjacent myofibers can be performed by using chemical inhibitors of signaling pathways, siRNAs, incubation with the respective signaling molecules, or overexpression using viral transduction [13-15] (see Note 1).

\section{Materials}

All materials for the isolation and culture of single myofibers need to be as sterile as possible. Therefore, we recommend performing dissection of the mouse and isolation of single myofibers under a semi-sterile dissection hood.

1. Tissue culture plates:

Coat all tissue culture plates (per mouse: $3-4$ wells of a 12-well plate for the isolation and 4-8 wells of a 24 -well plate for culturing) with sterile HS (horse serum) for approximately $5 \mathrm{~min}$. Remove the HS and let the plates dry for $5 \mathrm{~min}$.

2. Myofiber culture medium:

$20 \%$ FBS (fetal bovine serum), $1 \%$ chicken embryo extract in DMEM (Dulbecco's modified Eagle's medium; $4.5 \mathrm{~g} / \mathrm{l}$ glucose, $580 \mathrm{mg} / \mathrm{l} \mathrm{L}$-glutamine with $110 \mathrm{mg} / \mathrm{ml}$ sodium pyruvate), filter through $0.22 \mu \mathrm{m}$ filter before use. $30 \mathrm{~min}$ before starting the isolation, add the medium to the prepared 
tissue culture plates ( 24 well), and incubate them in a $37{ }^{\circ} \mathrm{C}$ incubator with $5 \% \mathrm{CO}_{2}$ to equilibrate the medium.

3. Myofiber isolation medium:

20\% FBS (fetal bovine serum) in DMEM (Dulbecco's modified Eagle's medium; $4.5 \mathrm{~g} / 1$ glucose, $580 \mathrm{mg} / 1$ L-glutamine with $110 \mathrm{mg} / \mathrm{ml}$ sodium pyruvate), filter through $0.22 \mu \mathrm{m}$ filter before use. $30 \mathrm{~min}$ before starting the isolation, add the medium to the prepared tissue culture plates (12 well), and incubate them in a $37{ }^{\circ} \mathrm{C}$ incubator with $5 \% \mathrm{CO}_{2}$ to equilibrate the medium.

4. Collagenase digestion solution:

$0.2 \%$ collagenase type I (Sigma \#C0130) in DMEM (Dulbecco's modified Eagle's medium; $4.5 \mathrm{~g} / \mathrm{l}$ glucose, $580 \mathrm{mg} / \mathrm{l}$ L-glutamine with $110 \mathrm{mg} / \mathrm{ml}$ sodium pyruvate), filter through $0.22 \mu \mathrm{m}$ filter before use. For two EDL (extensor digitorum longus) muscles $2.5 \mathrm{ml}$ of collagenase digestion solution are sufficient, transferred to a sterile $15 \mathrm{ml}$ reaction tube. Preheat the solution $10 \mathrm{~min}$ before starting the isolation in a $37^{\circ} \mathrm{C}$ circulating water bath ( see Note 2).

5. Dissection tools:

Fine forceps (Dumont 7, curved or straight)

Vannas spring scissors (cutting edge: $5 \mathrm{~mm}$, tip diameter: $0.35 \mathrm{~mm}$ )

Hardened fine curved scissors (cutting edge: $24 \mathrm{~mm}$ )

Fine forceps (Dumont 7b)

6. Stereo binocular microscope $(0.8-5 \times$ magnification $)$

7. Pipettes for dissociation of the muscles:

Prepare two kinds of sterile Pasteur pipettes: one large bore pipette for dissociation of the muscle and one small bore pipette for transfer of myofibers. Use a diamond pen to cut the glass Pasteur pipette to generate an opening of about $0.3 \mathrm{~cm}$, and heat polish to smoothen the pipette's edges. Also heat polish the small bore pipette. Flame to sterilize. Coat each pipette with HS before use.

8. Permeabilization buffer:

$0.1 \%$ Triton X-100, 0.1 M Glycine in PBS (pH 7.4)

9. Blocking solution for immunofluorescence:

5\% HS in PBS ( $\mathrm{pH} 7.4$ )

10. PFA:

2\% PFA in PBS ( $\mathrm{pH} 7.4)$

11. Antibodies for immunostaining:

Pax7 (PAX7, Developmental hybridoma bank, mouse IgGl; use undiluted)

MyoD (clone 5F11, Merck-Millipore, rat; dilution 1:100) 
Alexa Fluor 546 goat anti-mouse IgGl (Invitrogen; dilution 1:1000)

Alexa Fluor 488 goat anti-rat IgG (Invitrogen; dilution $1: 1000)$

12. DAPI staining solution:

$10 \mu \mathrm{g} / \mathrm{ml}$ DAPI $\left(4^{\prime}, 6\right.$-diamidino-2-phenylindole) in PBS ( $\mathrm{pH} 7.4)$

\section{Methods}

\subsection{Dissection and Digestion of the EDL Muscle}

1. Sacrifice the mouse according to animal welfare regulations (see Note 3 ).

2. Transfer the sacrificed mouse to a dissection bench (semisterile). Spray the whole mouse and dissection tools with $70 \%$ ethanol.

3. Remove the skin from the hind limb. Use forceps to lift up the skin at the ankle, and cut the skin with curved scissors up to the region over the knee, thereby exposing the underlying muscles (see Note 4; Fig. la). Make sure that no hairs are stuck to the exposed muscles, since they are the highest risk of contamination.

4. Remove the fascia surrounding the muscles by ripping them with fine forceps (Dumont 7). Pinch the fascia at the ankle at the side of the tibia bone with the forceps (close the forceps! Otherwise they will bend), and move the forceps toward the knee (Fig. 1b, c). The fascia will rip, and the tendon of the EDL (extensor digitorum longus) at the knee will be visible (Fig. ld).

5. Use curved fine forceps (Dumont 7) to expose the distal tendon of the TA (tibialis anterior) muscle (Fig. le; this is the tendon lying on top of the tendons at the ankle). Lift up the tendon with the forceps, and use another set of fine forceps to detach the TA muscle from the underlying EDL (extensor digitorum longus) muscle. Therefore, move the closed forceps up to the knee (approximately up to $0.2 \mathrm{~cm}$ below the knee) without injuring the EDL muscle.

6. Lift the tendon of the TA muscle with the fine forceps, and cut the tendon with fine spring scissors (Fig. lf). Pull the TA muscle up to the proximal end (Fig. lg); cut it at the knee or rip it off (Fig. lh). The EDL will be fully exposed now (Fig. li).

7. Grab the now fully exposed tendon of the EDL at the distal end (Fig. lj; see Note 5), cut the tendon with fine spring scissors (Fig. Ik), and pull the EDL muscle carefully toward the knee (Fig. 11). Do not touch the EDL muscle and do not stretch the muscle! Only handle the muscle at the tendon (see Note 6). 

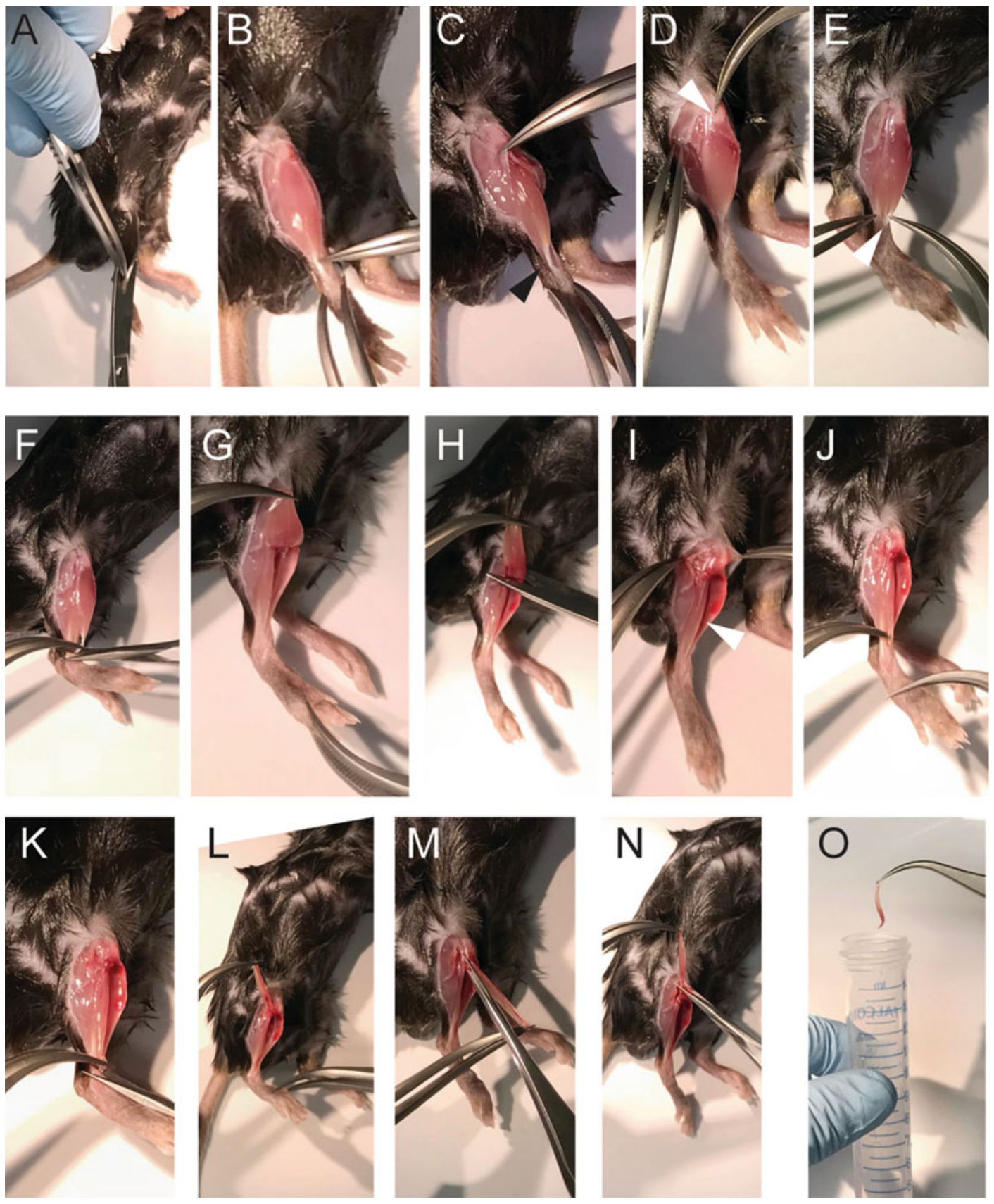

Fig. 1 Isolation of murine EDL muscle. (a) Hind limb of an adult mouse, (b) exposure of the TA muscle with its tendon, (c) removing of the fascia. (d) The proximal tendon of the EDL is now visible, marked by an arrowhead. (e-h) Removing of the TA muscle, (i) hind limb after removal of the TA muscle, the EDL muscle is now exposed, marked by an arrowhead. (j-0) Dissection and transfer of the EDL muscle to the collagenase digestion solution 

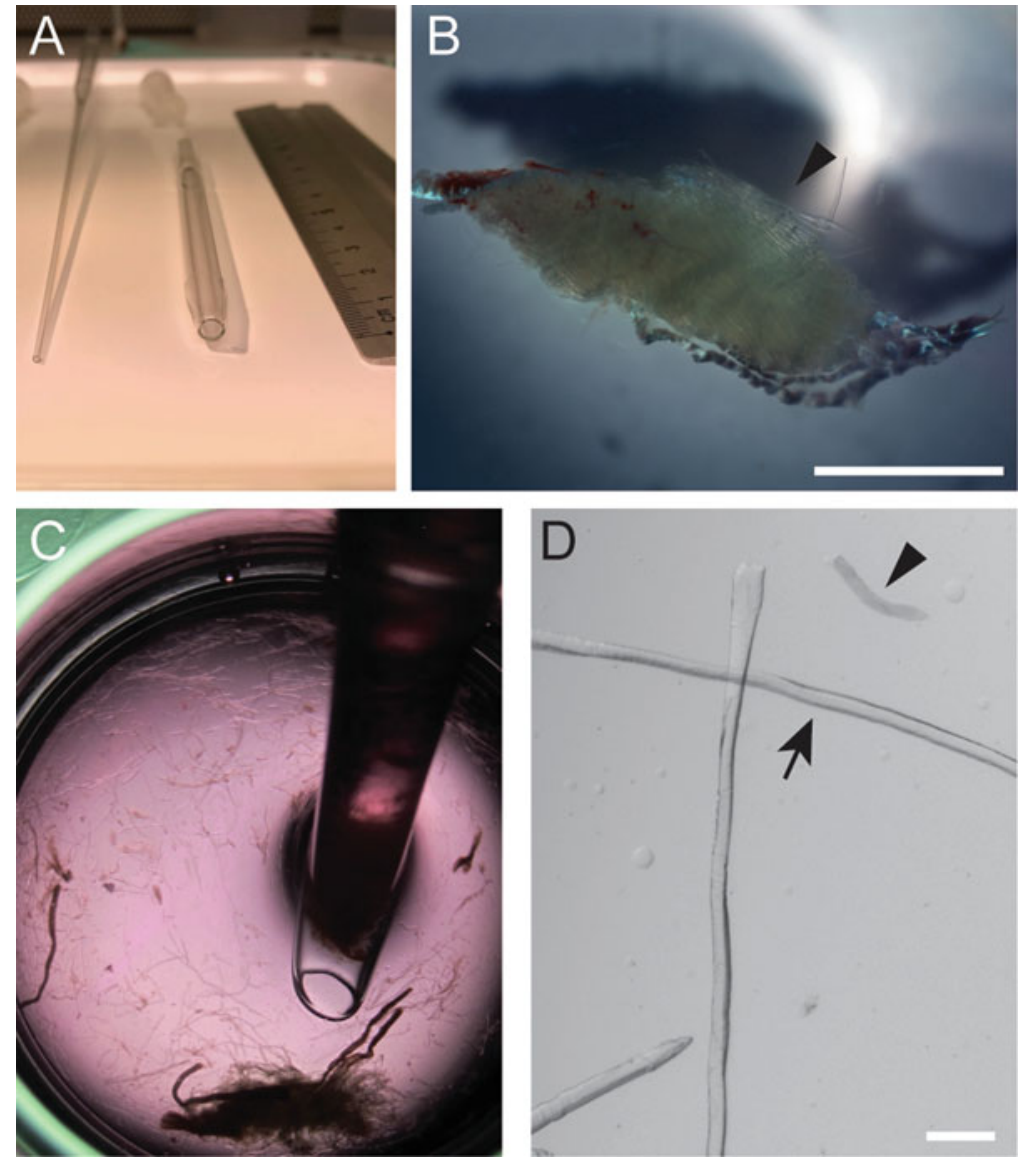

Fig. 2 Dissociation of the EDL muscle. (a) Small and large bore Pasteur pipettes for dissociation of the EDL muscle, (b) EDL muscle after $1 \mathrm{~h}$ digestion in collagenase digestion solution, (c) dissociation of the EDL muscle in a 12-well plate, (d) bright field image of isolated myofibers, the arrow marks a long intact alive myofiber, the arrowhead marks a short hyper contracted myofiber. Scale bar: $200 \mu \mathrm{m}$

8. At the knee there are two tendons visible (Fig. $1 \mathrm{~m}$ ). The tendon of the EDL is the one closer to the knee (see also Fig. 1d). Carefully pull the EDL toward the outside of the knee, and then cut the proximal tendon (Fig. In). Transfer the EDL gently to the preheated reaction tube containing the collagenase digestion solution (Fig. lo).

9. Repeat the procedure with the other leg.

10. Transfer the reaction tube with the two EDL muscles from one mouse into the $37{ }^{\circ} \mathrm{C}$ circulating water bath. Incubate until the myofibers are visible (Fig. 2b). The time of digestion is depending on collagenase activity, age of the mouse, and the amount of fibrosis. General digestion times are adult mouse 


\subsection{Dissociation of Single Myofibers}

\subsection{Immunostaining of MuSCs on Single Myofibers}

(2-6 months of age), $1 \mathrm{~h}$, and aged mouse ( 18 months and older), 1.5-2 h, depending on the amount of fibrotic tissue.

1. Transfer the digested EDL muscles into a well of a 12 -well plate filled with $2.5 \mathrm{ml}$ myofiber isolation medium (equilibrated in the incubator for about $30 \mathrm{~min}$ before use) using the large bore Pasteur pipette (Fig. 2a).

2. The next steps are done using a stereo binocular microscope with a 0.8-5-fold magnification under a dissection bench (semi-sterile) ( see Note 7).

3. Dissociate the muscles until single myofibers come off using the large bore Pasteur pipette (Fig. 2c; see Notes 8 and 9).

4. When about 50 myofibers have come off the muscle, transfer non-contracted single myofibers (shiny bright myofibers; Fig. 2d) into a new well of a 12 -well plate filled with equilibrated $2.5 \mathrm{ml}$ myofiber isolation medium. Transfer the myofibers with the small bore Pasteur pipette (Fig. 2a) releasing them gently into the myofiber isolation medium (see Notes 10-12).

5. Repeat Steps $\mathbf{3}$ and $\mathbf{4}$ until you have enough myofibers for your experiment (see Note 12).

1. Transfer about 50 non-contracted single myofibers into a well of a 24-well plate filled with $500 \mu \mathrm{l}$ of myofiber culture medium.

2. Culture the single myofibers in a $37{ }^{\circ} \mathrm{C}$ incubator with $5 \%$ $\mathrm{CO}_{2}$.

3. Transfection of MuSCs is done after $4 \mathrm{~h}$ of culture using Lipofectamine RNAiMAX: the final concentration of the siRNA equals 5 pmol. Add the reaction mix $(25 \mu$ l OptiMEM with the respective amount of siRNA in one reaction tube mixed with $25 \mu$ l OptiMEM and $1.5 \mu$ of Lipofectamine RNAiMAX, and then add the mixture to the $500 \mu \mathrm{l}$ of myofiber culture medium). It is not necessary to change the myofiber culture medium. For longer culture periods (over $48 \mathrm{~h}$ ), a second transfection after 24 h of culture might be considered.

1. Perform the immunostaining using a stereo binocular microscope with a $0.8-5$-fold magnification.

2. Fix the single myofibers with their adjacent MuSCs using $2 \%$ PFA for $5 \mathrm{~min}$ at room temperature. Therefore, remove the myofiber culture medium with a small bore pipette (HS coated) leaving a little bit of myofiber medium (about $150 \mu \mathrm{l}$ ) in the well to allow the myofibers to float in the medium. Then carefully add $500 \mu \mathrm{l}$ of PFA (2\%). Perform all further steps in a 24 well coated with HS (see Notes 13 and 14). 
3. Wash the myofibers three times with PBS $(500 \mu$ l per washing step, 5 min incubation time per washing step). Leave a little bit of solution in the 24 well to avoid sticking of the myofibers to the culture dish. Do this for all further steps unless stated otherwise.

4. Permeabilize the myofibers with permeabilization buffer $(500 \mu \mathrm{l})$ for $10 \mathrm{~min}$ at room temperature.

5. Block unspecific binding of antibodies by incubation with blocking solution $(500 \mu \mathrm{l}-1 \mathrm{ml})$ for $\mathrm{l} \mathrm{h}$ at room temperature.

6. Dilute the MyoD antibody (clone 5F11, rat, 1:100, MerckMillipore) in Pax7 antibody (PAX7 from Developmental hybridoma bank, mouse IgGl, undiluted), use $250 \mu \mathrm{l}$ per well of a 24-well plate, and incubate overnight at $4^{\circ} \mathrm{C}$.

7. Wash three times with PBS at room temperature $(5 \mathrm{~min}$ per washing step).

8. Incubate with secondary antibodies $(250 \mu \mathrm{l}$ per well, incubation for $\mathrm{l} \mathrm{h}$ at room temperature in the dark, therefore use tin foil to wrap the culture plate). Dilute Alexa Fluor 546 goat anti-mouse IgGl specific antibody and Alexa Fluor 488 goat anti-rat antibody in blocking solution (1:1000). Every following step should be done under light reduced conditions.

9. Wash twice with PBS at room temperature $(5 \mathrm{~min}$ per washing step).

10. Perform DAPI staining $(500 \mu \mathrm{l}$ per well, final concentration: $10 \mu \mathrm{g} / \mathrm{ml}$ ) for $5 \mathrm{~min}$ at room temperature.

11. Wash twice with PBS at room temperature $(5 \mathrm{~min}$ per washing step).

12. During the final washing steps, label the glass microscope slides on which the myofibers will be mounted. A PAP pen can be used to draw a hydrophobic circle around the edges of the glass slides, thereby avoiding spilling of myofiber containing liquid over the edges of the slide.

13. After the final washing step, transfer the stained myofibers to the glass microscope slide in the smallest volume possible. Make sure the single myofibers are spread out on the glass microscope slide, so you can count the MuSCs on each myofiber separately.

14. Remove the liquid with a $200 \mu$ pipette. Make sure that the myofibers are not dragged over the slide; rather leave a little bit of liquid on the slide.

15. Add two to three drops of mounting medium, and apply a cover slip, thereby avoiding the generation of air bubbles (see Note 15). 
16. Let the slides dry at room temperature for at least 20-60 min before analyzing them at the microscope. Make sure that the cover slip is not moving on the slide when counting the cells using a fluorescence microscope. If necessary let the mounting medium harden over night at $4{ }^{\circ} \mathrm{C}$ ( see Notes 16-18).

\section{Notes}

1. Also fix some myofibers with their adjacent MuSCs directly after isolation. This gives you a reference for the number of MuSCs per myofiber before culture.

2. Always prepare the collagenase digestion solution on the day of myofiber isolation.

3. Also perform cervical dislocation. This results in bleeding at the neck and less bleeding after cutting the TA muscle.

4. Make sure that the dissection is done as sterile as possible; otherwise the risk of contamination is quite high. We recommend using two sets of forceps and scissors, one for cutting the fur and one for cutting the muscles. That minimizes the risk of contamination.

5. Make sure that you handle the EDL muscles only at the tendons; otherwise the muscle will contract and the myofibers will die.

6. If the EDL muscle is ripping when pulling it toward the knee, you have grabbed the distal tendon at the location after it branches. Use your free hand to grab the part of the tendon you missed at the foot. Loosen it and grab both tendon parts with one forceps and continue the dissection.

7. Using a heated plate for keeping the myofibers warm when dissociating them increases the overall survival of myofibers, especially for myofibers isolated from aged mice.

8. If the opening of the large bore pipette is too wide, the myofibers will not come apart. Try with a smaller one.

9. If more than $30 \%$ of your isolated myofibers are hyper contracted, the force applied is too high. Either try dissociating with less force or use a large bore pipette with a bigger opening.

10. An additional washing step in a 12 -well plate filled with myofiber isolation medium $(2.5 \mathrm{ml})$ decreases sticking together of myofibers.

11. Isolation of myofibers from two EDL muscles should give enough myofibers for analyzing 5-6 conditions (with 50 myofibers per condition each). 

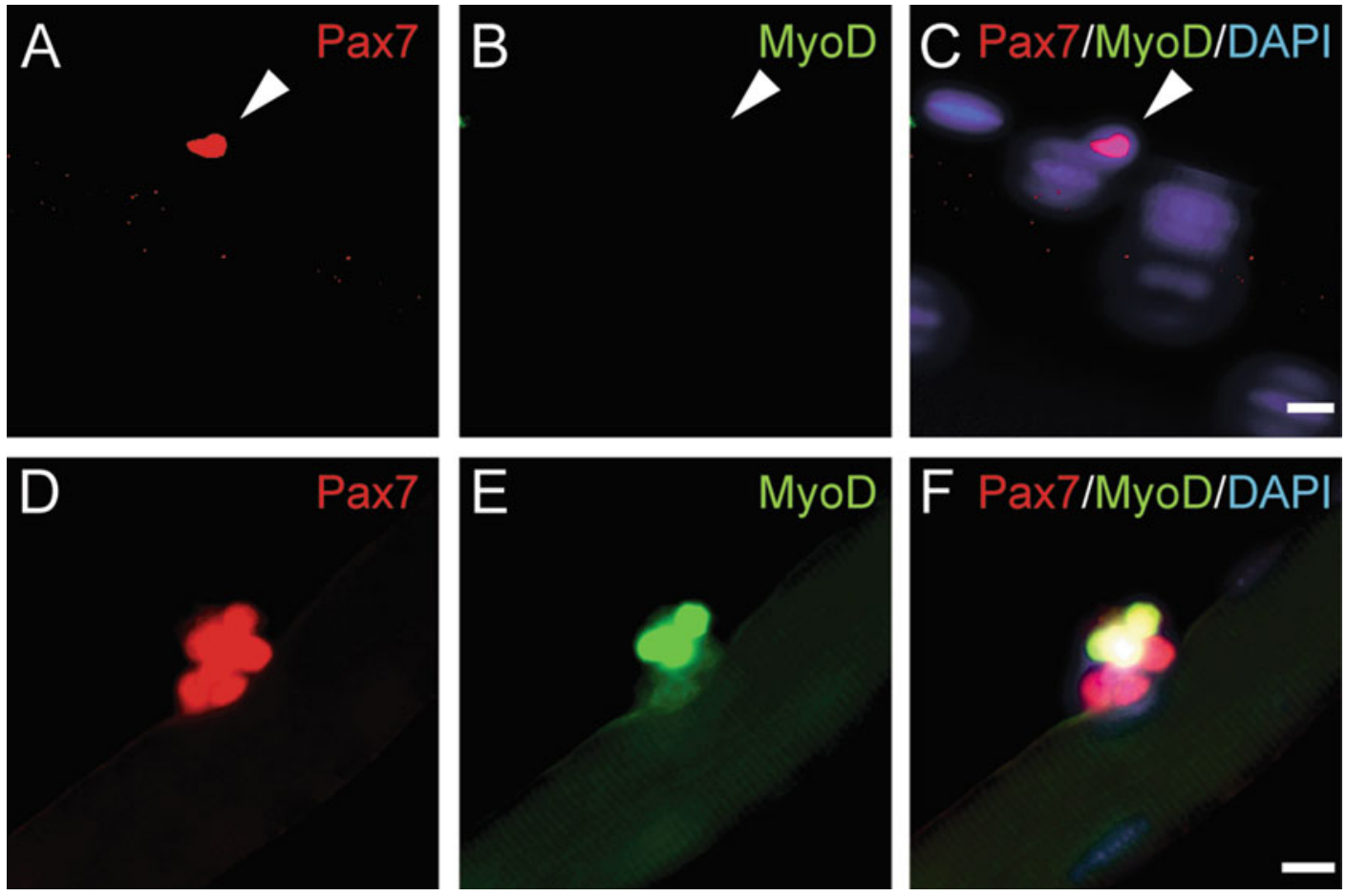

Fig. 3 Immunostaining of isolated myofibers with their adjacent MuSCs. Representative immunostaining of a MuSC on its adjacent myofiber fixed directly after isolation or a cluster of MuSCs after $72 \mathrm{~h}$ of culture. Staining with antibodies directed to $\operatorname{Pax} 7(\mathbf{a}, \mathbf{d}), \operatorname{MyoD}(\mathbf{b}, \mathbf{e})$, and DAPI (c, f). The arrowhead in a-c denotes the MuSC. Scale bar: $10 \mu \mathrm{m}$

12. Make sure that you start dissociating the myofibers only when you see the first myofibers coming off the digested muscle. Otherwise incubate longer.

13. MuSCs on their adjacent myofibers can be cultivated up to $96 \mathrm{~h}$ under floating conditions. The transfer of isolated myofibers into the 24 well containing the myofiber culture medium is regarded as $0 \mathrm{~h}$.

14. Myofibers isolated from aged mice have a higher tendency to attach to each other during culture than myofibers isolated from young animals. To dissociate them you can add the PBS for washing after the fixation with a little bit more force than normally. Make sure that you do not wash the clusters of MuSCs off the myofiber.

15. Check that the staining was successful before mounting all myofibers. Therefore take out a few myofibers, mount them, and check at the microscope for successful staining (Fig. 3). It is not necessary to wait for the mounting medium to harden for this test. 
16. If there are only very few clusters per myofiber formed (less than five for young animals and less than two or three for old animals), this can have multiple reasons: the clusters might have been washed off or might have been ripped off during mounting of the myofibers. It is also possible that the myofiber culture medium is not containing adequate amounts of growth factors. Try a new batch of chicken embryo extract or FBS.

17. Dead myofibers can be easily identified under the light microscope since they are hyper contracted and very short (Fig. $2 \mathrm{~d}$ ).

18. Adding antibiotics to the myofiber isolation and culture medium will affect the MuSCs. Therefore, it is not advisable.

\section{Acknowledgments}

This work was supported by a grant from the DFG to J.v.M (MA-3975/2-1). We would like to thank Christine Poser and Christina Picker for excellent technical assistance.

\section{References}

1. Lepper C, Partridge TA, Fan CM (2011) An absolute requirement for Pax7-positive satellite cells in acute injury-induced skeletal muscle regeneration. Development 138 (17):3639-3646. https://doi.org/10.1242/ dev.067595

2. Murphy MM, Lawson JA, Mathew SJ, Hutcheson DA, Kardon G (2011) Satellite cells, connective tissue fibroblasts and their interactions are crucial for muscle regeneration. Development 138(17):3625-3637. https://doi.org/10.1242/dev.064162

3. Bentzinger CF, von Maltzahn J, Rudnicki MA (2010) Extrinsic regulation of satellite cell specification. Stem Cell Res Ther 1(3):27. https://doi.org/10.1186/scrt27

4. Shea KL, Xiang W, LaPorta VS, Licht JD, Keller C, Basson MA, Brack AS (2010) Sproutyl regulates reversible quiescence of a selfrenewing adult muscle stem cell pool during regeneration. Cell Stem Cell 6(2):117-129. https://doi.org/10.1016/j.stem.2009.12. 015

5. von Maltzahn J, Bentzinger CF, Rudnicki MA (2013) Characteristics of satellite cells and multipotent adult stem cells in the skeletal muscle. Stem cells and cancer stem cells. Springer, 12:63-73

6. Bentzinger CF, Wang YX, Rudnicki MA (2012) Building muscle: molecular regulation of myogenesis. Cold Spring Harb Perspect Biol
4(2). https://doi.org/10.1101/cshperspect. a008342

7. Bernet JD, Doles JD, Hall JK, Kelly Tanaka K, Carter TA, Olwin BB (2014) p38 MAPK signaling underlies a cell-autonomous loss of stem cell self-renewal in skeletal muscle of aged mice. Nat Med 20(3):265-271. https://doi. org $/ 10.1038 / \mathrm{nm} .3465$

8. Price FD, von Maltzahn J, Bentzinger CF, Dumont NA, Yin H, Chang NC, Wilson DH, Frenette J, Rudnicki MA (2014) Inhibition of JAK-STAT signaling stimulates adult satellite cell function. Nat Med 20(10):1174-1181. https://doi.org/10.1038/nm.3655

9. Tierney MT, Aydogdu T, Sala D, Malecova B, Gatto S, Puri PL, Latella L, Sacco A (2014) STAT3 signaling controls satellite cell expansion and skeletal muscle repair. Nat Med 20 (10):1182-1186. https://doi.org/10.1038/ nm.3656

10. Schworer S, Becker F, Feller C, Baig AH, Kober U, Henze $\mathrm{H}$, Kraus JM, Xin B, Lechel A, Lipka DB, Varghese CS, Schmidt M, Rohs R, Aebersold R, Medina KL, Kestler HA, Neri F, von Maltzahn J, Tumpel S, Rudolph KL (2016) Epigenetic stress responses induce muscle stem-cell ageing by Hoxa9 developmental signals. Nature 540 (7633):428-432. https://doi.org/10.1038/ nature 20603 
11. Sousa-Victor P, Gutarra S, Garcia-Prat L, Rodriguez-Ubreva J, Ortet L, Ruiz-Bonilla V, Jardi M, Ballestar E, Gonzalez S, Serrano AL, Perdiguero E, Munoz-Canoves P (2014) Geriatric muscle stem cells switch reversible quiescence into senescence. Nature 506 (7488):316-321. https://doi.org/10.1038/ naturel3013

12. von Maltzahn J, Chang NC, Bentzinger CF, Rudnicki MA (2012) Wnt signaling in myogenesis. Trends Cell Biol 22(11):602-609. https://doi.org/10.1016/j.tcb.2012.07.008

13. Pasut A, Jones AE, Rudnicki MA (2013) Isolation and culture of individual myofibers and their satellite cells from adult skeletal muscle. $\mathrm{J}$ Vis $\operatorname{Exp~(73):e50074.~https://doi.org/10.~}$ $3791 / 50074$

14. Bentzinger CF, Wang YX, von Maltzahn J, Soleimani VD, Yin H, Rudnicki MA (2013) Fibronectin regulates Wnt7a signaling and satellite cell expansion. Cell Stem Cell 12 (1):75-87. https://doi.org/10.1016/j.stem. 2012.09.015

15. von Maltzahn J, Zinoviev R, Chang NC, Bentzinger CF, Rudnicki MA (2013) A truncated Wnt7a retains full biological activity in skeletal muscle. Nat Commun 4:2869. https://doi.org/10.1038/ncomms3869

Open Access This chapter is licensed under the terms of the Creative Commons Attribution 4.0 International License (http://creativecommons.org/licenses/by/4.0/), which permits use, sharing, adaptation, distribution and reproduction in any medium or format, as long as you give appropriate credit to the original author(s) and the source, provide a link to the Creative Commons license and indicate if changes were made.

The images or other third party material in this chapter are included in the chapter's Creative Commons license, unless indicated otherwise in a credit line to the material. If material is not included in the chapter's Creative Commons license and your intended use is not permitted by statutory regulation or exceeds the permitted use, you will need to obtain permission directly from the copyright holder. 